\title{
How can the accuracy of SEEG be increased? - an analysis of the accuracy of multilobe-spanning SEEG electrodes based on a frameless stereotactic robot-assisted system
}

\author{
Chao Lu ${ }^{1,2 \#}$, Sichang Chen ${ }^{1,2 \#}$, Yang An ${ }^{1,2}$, Fei Meng ${ }^{1,2}$, Yihe Wang ${ }^{1,2}$, Penghu Wei ${ }^{1,2}$, Xiaotong Fan ${ }^{1,2}$, \\ Yongzhi Shan ${ }^{1,2}$, Guoguang Zhao ${ }^{1,2,3}$ \\ ${ }^{1}$ Department of Neurosurgery, Xuanwu Hospital, Capital Medical University, Beijing, China; ${ }^{2}$ China International Neuroscience Institute (CHINA- \\ INI), Beijing, China; ${ }^{3}$ Center of Epilepsy, Beijing Institute for Brain Disorders, Beijing, China \\ Contributions: (I) Conception and design: Y Shan; G Zhao; (II) Administrative support: G Zhao; (III) Provision of study materials or patients: C Lu; S \\ Chen; (IV) Collection and assembly of data: Y An; F Meng; Y Wang; (V) Data analysis and interpretation: P Wei, X Fan; (VI) Manuscript writing: All \\ authors; (VII) Final approval of manuscript: All authors. \\ \#These authors contributed equally to this work. \\ Correspondence to: Yongzhi Shan; Guoguang Zhao. Department of Neurosurgery, Xuanwu Hospital, Capital Medical University, No. 45, Changchun \\ Street, Xicheng District, Beijing 100053, China. Email: shanyongzhi@xwhosp.org; ggzhao@vip.sina.com.
}

Background A frameless stereotactic robot-assisted system allows stereoelectroencephalography (SEEG) electrodes to span multiple lobes. As the angularity and length are increased, maintaining accuracy of the electrodes becomes more challenging. The goal of this study was to analyze the factors that influence the accuracy of multilobe-spanning SEEG electrodes inserted using a frameless stereotactic robot-assisted system.

Methods: A total of 322 SEEG electrodes were implanted in 39 patients with refractory epilepsy, and sixtyone multilobe-spanning SEEG electrodes were selected to analyze the factors that influenced the accuracy of implantation. The target error, entrance error, depth error, and angular error were calculated by a specialized computer program. Factors including electrode depth, angular deviation, referencing method, head holder choice, and use of a predrill procedure were analyzed to determine their effects on accuracy.

Results: Thirty-nine patients (aged 2-35 years, median: 19 years; 21 females) underwent frameless robotassisted SEEG electrode implantation. The mean distance between the intended target and actual tip location was $2.57 \pm 1.70 \mathrm{~mm}$ (range, $0.42-9.02 \mathrm{~mm}$ ). The mean distance between the intended entrance point and the actual location was $2.2 \pm 1.29 \mathrm{~mm}$ (range, $0.70-6.13 \mathrm{~mm}$ ). The mean length of the electrodes was $84.63 \pm 7.61 \mathrm{~mm}$ (range, 70.60-103.99 mm). The depth error was $1.36 \pm 1.22 \mathrm{~mm}$ (range, 0.03-6.69 mm), and the angular deviation was 1.64 \pm 1.12 degrees (range, 0.15-4.93 degrees). Multifactor regression analysis showed that entrance error, electrode depth, depth error, angular deviation, referencing method, and head holder choice could explain 59.5\% of the electrode target error. Angular deviation, choice of registration approach and head holder and the use of a predrill procedure could explain $48.1 \%$ of the electrode entrance error. Use of a predrill procedure significantly reduced the electrode angular deviation $(\mathrm{P}<0.05)$.

Conclusions: Head holder choice, use of a predrill procedure and angular deviation are the primary influencing factors of the accuracy of multilobe-spanning SEEG electrode placement. The Leksell frame and a predrill procedure can be used to increase the accuracy of SEEG electrode placement.

Keywords: Stereoelectroencephalography (SEEG); Robotic Stereotactic Assistance (ROSA); influencing factors; epilepsy; multilobe-spanning electrode

Submitted Oct 27, 2020. Accepted for publication Dec 24, 2020.

doi: 10.21037/apm-20-2123

View this article at: http://dx.doi.org/10.21037/apm-20-2123 


\section{Introduction}

Stereoelectroencephalography (SEEG) is widely used for surgical evaluation of epilepsy patients whose epileptogenic zone cannot be located by a noninvasive assessment approach (1-3). The safety and effectiveness of SEEG electrodes depend on accurate implantation. With the development of frameless stereotactic robot-assisted system technology, the SEEG electrode angularity and length can be increased to span multiple lobes. However, compared with that of conventional electrodes, the implantation of long-spanning electrodes is highly challenging, especially for high skew-angle trajectories (more than 30 degrees from classical orthogonal trajectories). This can lead to a larger error in the accuracy, as oblique trajectories, often used for insular lobe (4), hippocampus (5), or hypothalamic hamartoma (6), could lead to the drill sliding at the entrance of the skull and a subsequent error in the direction of the electrode. The accuracy of SEEG not only influences the safety of the procedure but also could be related to the therapeutic efficacy because oblique, longspanning electrodes are often designed for radiofrequency thermocoagulation. We report the results of a quantitative, multiple influencing factor analysis of the accuracy of 61 multilobe-spanning SEEG electrodes in a subset of 39 patients.

We present the following article in accordance with the STROBE reporting checklist (available at http://dx.doi. org/10.21037/apm-20-2123).

\section{Methods}

\section{Patients}

We retrospectively reviewed the medical and imaging data of 39 consecutive patients who received multilobe-spanning SEEG electrode implantation due to intractable epilepsy from January 2017 to January 2020 at Xuanwu Hospital by a single surgeon. A total of 322 SEEG electrodes were implanted. Noninvasive investigations, including scalp electroencephalography, magnetic resonance imaging (MRI) and semiology analysis, were performed for all patients, which indicated the diagnosis of focal epilepsy and candidates for SEEG implantation. All patients underwent SEEG electrode implantation procedures for intracranial electrophysiological monitoring and minimally invasive treatment after a multidisciplinary team (MDT) evaluation.

The inclusion criteria were as follows: (I) multilobespanning electrodes; (II) the electrode length exceeded
$70 \mathrm{~mm}$; (III) the electrodes were implanted with the Robotic Stereotactic Assistance (ROSA) system. The exclusion criteria were single lobe electrodes and electrodes less than $70 \mathrm{~mm}$. Sixty-one multilobe-spanning SEEG electrodes were finally included in the present study.

The study was conducted in accordance with the Declaration of Helsinki (as revised in 2013). The study was approved by the ethics committee at Xuanwu Hospital (No. LYS [2019] 097) and informed consent was taken from all the patients.

\section{Implantation of SEEG electrodes}

The SEEG electrode implantation strategy was determined by the MDT, which included neurosurgeons, neurologists, neuroradiologists and electrophysiologists specializing in epilepsy. SEEG electrodes with a diameter of $0.8 \mathrm{~mm}$ and 5-18 contacts (Alcis, Besancon, France) were used in our center. A three-dimensional magnetization-prepared rapid acquisition gradient echo (MPRAGE) sequence was carried out for presurgical SEEG electrode trajectory planning via ROSA software, and a thin-slice computed tomography (CT) scan was performed, with or without scalp fiducials attached to the head, for robotic registration.

The electrodes were implanted under general anesthesia via the following surgical procedure. The head was positioned by a Mayfield clamp or Leksell frame as the head holder, which was fixed with the ROSA system. Next, laser facial scanning or scalp fiducial markers were registered by the robotic system. Then, the skin and skull were drilled with a regular drill; a predrill procedure involving a thick, flat-tip drill was used to grind a platform onto the skull to avoid trajectory deviation for electrodes with a planned angle of less than 60 degrees with respect to the skull surface (details in another paper, unpublished). After attaching the bolt, the dura was ruptured by a needle and a blunt guide to establish a tunnel for electrode implantation.

\section{Measurement of SEEG electrode implantation accuracy}

A thin-slice CT scan was performed within 1 day after implantation and was then fused with the preoperative CT scan using ROSA software. The electrode position on the postoperative CT scan was then compared with the planned trajectory on the preoperative MPRAGE images. The midcommissural point (MCP) was labeled for all patients as the coordinate origin $(X=Y=Z=0)$, which was used as the reference point to determine the Cartesian coordinates 
Table 1 A summary of multilobe-spanning electrode accuracy $(n=61)$

\begin{tabular}{lcccc}
\hline Variable & Mean & SD & Min & Max \\
\hline Target error $(\mathrm{mm})$ & 2.57 & 1.70 & 0.42 & 9.02 \\
Entry error $(\mathrm{mm})$ & 2.2 & 1.29 & 0.70 & 6.13 \\
Depth $(\mathrm{mm})$ & 84.63 & 7.61 & 70.60 & 103.99 \\
Depth error $(\mathrm{mm})$ & 1.36 & 1.22 & 0.03 & 6.69 \\
Angular deviation (degrees) & 1.64 & 1.12 & 0.15 & 4.93 \\
\hline
\end{tabular}

Table 2 Choice of particular frames and registration approaches and the use of a predrill procedure

\begin{tabular}{lc}
\hline Variable & $\mathrm{n}(\%)$ \\
\hline Head holder & $30(50.82)$ \\
Leksell frame & $31(49.18)$ \\
Mayfield clamp & \\
Registration approach & $48(78.69)$ \\
Laser facial scanning & $13(21.31)$ \\
Scalp fiducials & \\
Predrill procedure & $37(60.66)$ \\
Yes & $24(39.34)$ \\
No &
\end{tabular}

of the tips and entry points of both the planning and actual electrodes. We defined the coordinates of the actual target point as $(\mathrm{X} 0, \mathrm{Y} 0, \mathrm{Z} 0)$, the actual entry point as (X1, Y1, Z1), the planning target point as (X2, Y2, Z2), and the planning entry point as $(\mathrm{X} 3, \mathrm{Y} 3, \mathrm{Z} 3)$. The entry points of the electrodes were selected as the point at which they intersected the inner plate of the skull. The Euclidean distances between two points were calculated using the formula: $\sqrt{(X a-X b) 2+(Y a-Y b) 2+(Z a-Z b) 2}$. The angular deviation was calculated as the intersection of the lines corresponding to the planning electrode and the actual electrode.

\section{Statistical analysis}

Statistical analysis was performed using SPSS (version 25). The effects of entrance error, electrode depth, depth error, angular deviation, registration approach, and head holder choice on target error were analyzed by linear regression. Additionally, the effects of angular deviation, registration approach, head holder choice and predrill procedure on entrance error were analyzed by linear regression.

\section{Results}

Among the 322 electrodes implanted in 39 patients, 61 trajectories were included because the length was more than $70 \mathrm{~mm}$. The mean distance between the intended target and actual tip location was $2.57 \pm 1.70 \mathrm{~mm}$ (range, $0.42-9.02 \mathrm{~mm}$ ). The mean distance between the intended entrance point and the actual location was $2.2 \pm 1.29 \mathrm{~mm}$ (range, $0.70-6.13 \mathrm{~mm}$ ). The mean length of the electrodes was $84.63 \pm 7.61 \mathrm{~mm}$ (range, $70.60-103.99 \mathrm{~mm}$ ). The mean depth error and angular deviation were $1.36 \pm 1.22 \mathrm{~mm}$ (range, $0.03-6.69 \mathrm{~mm}$ ) and $1.64 \pm 1.12$ degrees (range, $0.15-$ 4.93 degrees), respectively (Table 1). The number of times that a particular head holder or registration approach was chosen and that a predrill procedure was used are shown in Table 2. Among this patient series, no neurological deficits or intracranial hemorrhage were found to have been caused by the implantation.

The effect on the target error of the entrance error, electrode depth, depth error, and angular deviation and the effect on the entrance error of the angular deviation are shown in Figure 1. The target error exhibited a significant positive correlation with the entrance error $\left(R^{2}=0.443\right.$, $\mathrm{P}<0.01)$, depth error $\left(\mathrm{R}^{2}=0.238, \mathrm{P}<0.01\right)$, and angular deviation $\left(\mathrm{R}^{2}=0.351, \mathrm{P}<0.05\right)$ but was not significantly correlated with the electrode depth $\left(\mathrm{R}^{2}=0.0006, \mathrm{P}=0.269\right)$ or the choice of registration approach $(\mathrm{P}=0.579)$ or head holder $(\mathrm{P}=0.511)$ (Table 3). The entrance error exhibited a significant positive correlation with the angular deviation $\left(\mathrm{R}^{2}=0.347, \mathrm{P}<0.01\right)$ and a negative correlation with the use of a predrill procedure $(\mathrm{P}<0.05)$; it also had a significant correlation with the head holder choice $(\mathrm{P}<0.05$; the Leksell frame was superior to the Mayfield clamp) but not with the registration approach $(\mathrm{P}=0.565)$ (Table 4). Independent 

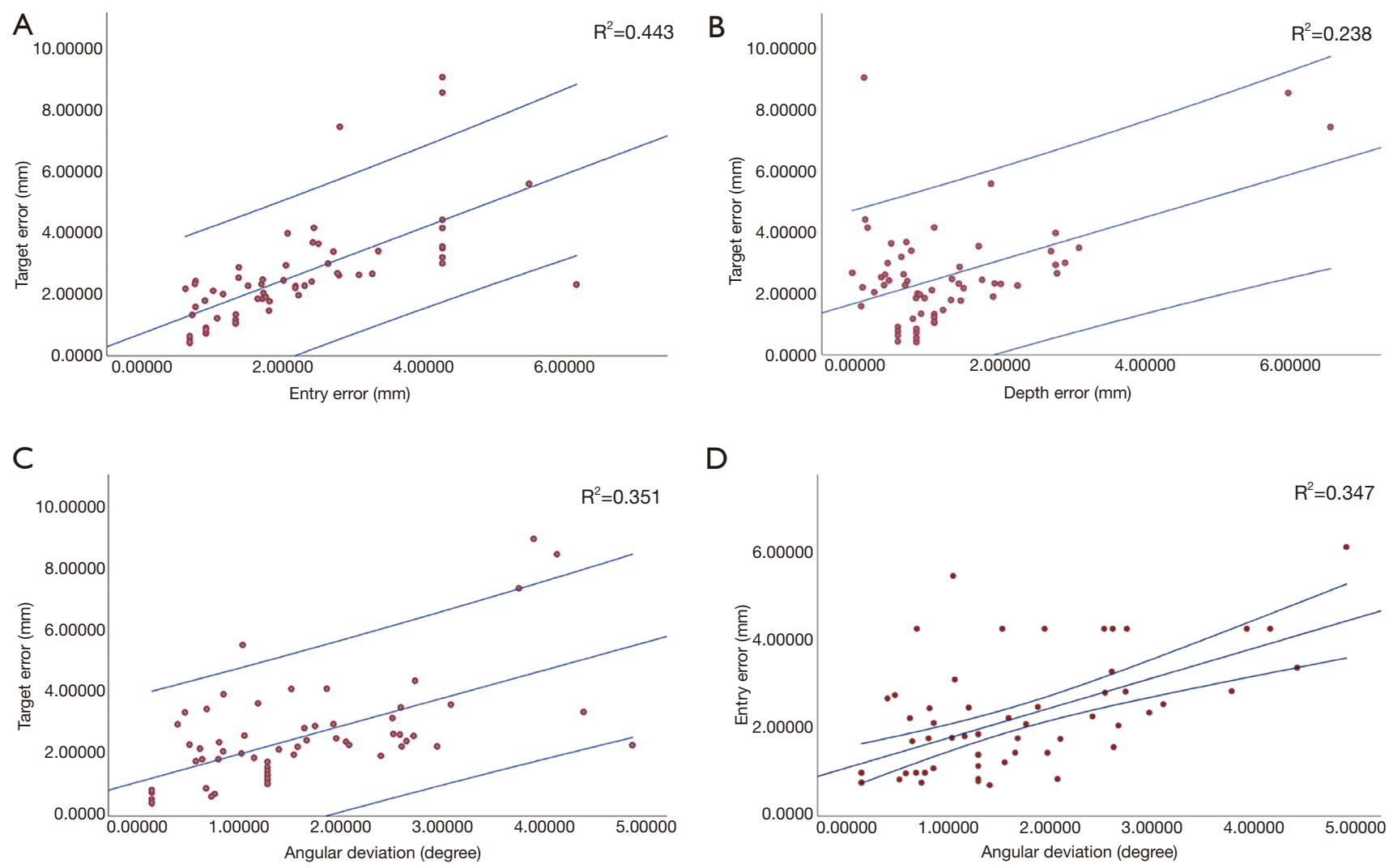

Figure 1 The statistical analysis results. (A) Entry error had a significant positive correlation with the target error $\left(R^{2}=0.443, P=0.001\right)$ with a slope of 0.88 and intercept of 0.63 . (B) Depth error had a significant positive correlation with the target error $\left(\mathrm{R}^{2}=0.238, \mathrm{P}=0.003\right)$ with a slope of 0.67 and intercept of 1.67. (C) Angular deviation had a significant positive correlation with the target error $\left(\mathrm{R}^{2}=0.351, \mathrm{P}=0.037\right)$ with a slope of 0.9 and intercept of 1.09 . (D) Angular deviation had a significant positive correlation with the entry error $\left(\mathrm{R}^{2}=0.347, \mathrm{P}=0.001\right)$ with a slope of 0.68 and intercept of 1.09 .

Table 3 Regression analysis of influencing factors of the target error for multilobe-spanning electrodes

\begin{tabular}{lcccc}
\hline Variable & B & Beta & t & P \\
\hline Entry error & 0.564 & 0.428 & 3.634 & 0.001 \\
Depth & -0.022 & -0.107 & -1.226 & 0.269 \\
Depth error & 0.403 & 0.292 & 3.074 & 0.003 \\
Angular deviation & 0.363 & 0.239 & 2.137 & 0.037 \\
Registration approach & 0.213 & 0.052 & 0.559 & 0.579 \\
Head holder choice & -0.216 & -0.064 & -0.662 & 0.511 \\
\hline
\end{tabular}

Model $R^{2}=0.595$, adjusted $R^{2}=0.551, F=13.249$, and $P<0.05$. 
Table 4 Regression analysis of influencing factors of the entrance error for multilobe-spanning electrodes

\begin{tabular}{lcccc}
\hline Variable & $\mathrm{B}$ & Beta & $\mathrm{t}$ & $\mathrm{P}$ \\
\hline Angular deviation & 0.608 & 0.528 & 5.371 & 0.000 \\
Registration approach & 0.182 & 0.058 & 0.579 & 0.565 \\
Head holder choice & -0.053 & -0.207 & -2.047 & 0.045 \\
Use of a predrill procedure & -0.071 & -0.272 & -2.648 & 0.010 \\
\hline
\end{tabular}

Model $R^{2}=0.481$, adjusted $R^{2}=0.444, F=12.979$, and $P<0.05$.
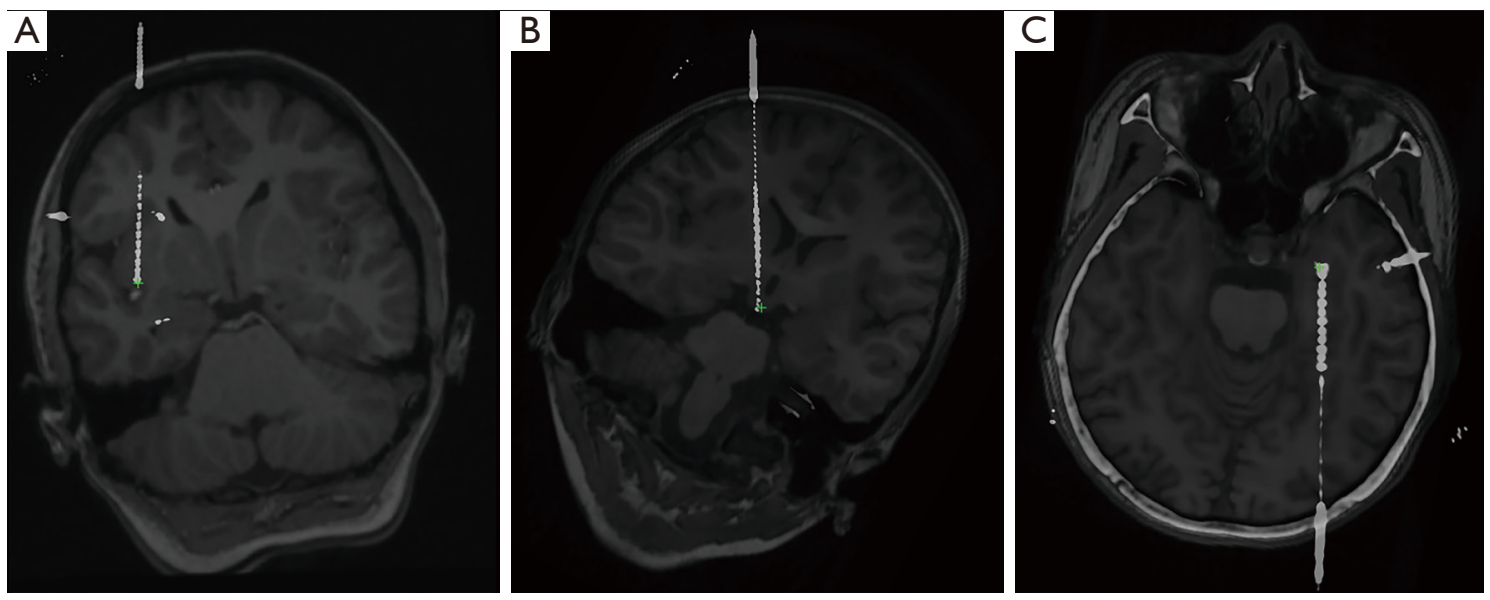

Figure 2 Examples of implanted multilobe-spanning electrodes. (A) Frontal-insular lobe electrode. (B) Frontal-basal ganglia-hypothalamic hamartoma electrode. (C) Occipital-hippocampus long-axis electrode.

sample $t$-tests showed that using a predrill procedure was a protective factor for the electrode angular deviation $(\mathrm{P}<0.05)$.

\section{Discussion}

The present study revealed the accuracy of multilobespanning SEEG and analyzed the possible influencing factors, including the entrance error, electrode depth, depth error, angular deviation, surgical procedure, registration approach, and head holder selection, aiming to improve the surgical technique to increase the accuracy and safety of SEEG electrode implantation.

The development of stereotaxy can be dated back 100 years, and frameless stereotaxy has been shown to be comparable to frame-based stereotaxy in terms of accuracy, diagnostic yield, morbidity, and mortality $(7,8)$. However, the craniotomy direction and angle are more flexible in frameless stereotaxy than those in frame-based stereotaxy. The SEEG electrode can span multiple lobes using a frameless stereotactic robot-assisted system with a direction and angle suitable for clinical needs (Figure 2). Nevertheless, compared with conventionally-implanted electrodes, accurately implanting SEEG electrodes is challenging, especially for high skew-angle trajectories (more than 30 degrees). This can lead to a larger error in the accuracy because an oblique trajectory often leads to the drill sliding at the entrance of the skull and a subsequent error in the direction of the electrode (9).

Accuracy is the key to depth electrode implantation. Complications could arise from deviations in electrode placement, such as a failure to localize the seizure onset zone or injury to blood vessels and important functional areas (10). In the present study, we analyzed the factors that influenced the accuracy of multilobe-spanning SEEG electrodes implanted using a frameless stereotactic robotassisted system to offer a methodology to improve electrode placement accuracy. Target errors were mainly caused by entrance errors, angular errors, depth errors and direction deviations. In the present study, we showed that the target 
error exhibited significant correlations with the entrance error, depth error, and angular error by multifactor linear regression, and thus deviation in the direction of the electrode was excluded from the subsequent statistics. Therefore, to control the target error, measures should be taken to minimize the effects of influencing factors. None of the techniques for SEEG electrode implantation directly affected the depth error, as this is controlled by the surgeon. It is necessary to perform accurate calculations to the decimeter/millimeter level and have them doublechecked by two medical staff members. Iordanou et al. noted that SEEG trajectories with a planned angle of $>30$ degrees had significantly higher radial errors than trajectories with a planned angle of $<30$ degrees (9). They suggested lowering the robotic sleeve slowly until it pinches the skin slightly to decrease the distance from the robotic guide to the target. Additionally, when drilling is initiated, very slight pressure should be applied initially to allow the drill to penetrate the outer cortex of the skull. In our previous study, we developed a predrill procedure that uses a thick, flat-tip drill to grind a platform onto the skull surface before burr hole drilling. The analysis showed that the predrill procedure could significantly reduce deviations in the electrode angle.

Entrance errors were mainly caused by angular deviations and the choice of the stereotaxic system and registration approach. In a single-center study, Girgis et al. demonstrated that frame-based SEEG implantation is significantly more accurate and precise and results in more clinically useful electrode contacts than frameless implantation using a navigation guidance system (11). Cardinale et al. demonstrated a significant improvement in the SEEG electrode accuracy with the NeuroMate robotic system than with the Talairach frame (8). A similar study showed that the entrance error was not significantly different between the ROSA robotic system and the Leksell frame (12). In the present study, laser facial scanning registration did not significantly improve the entrance accuracy. Bone fiducials may lead to high registration accuracy due to their stability (13). A retrospective study demonstrated that the bone fiducial registration approach did not significantly improve the target error, entry error or angular deviation over laser facial scanning registration (14). The authors concluded that this was due to the use of high-definition CT scans as registration images and of bone fiducials as posterior trajectories, which may affect the accuracy. In our center, laser facial scanning registration is commonly used in clinical practice. However, the facial expression during the CT scan and the pull of the frame nail on the scalp may affect the accuracy of registration. The patients in our center are required to maintain neutral facial expressions and to not wear anything, including a hat or mask, during the CT scan. According to the present study, the choice of head holder resulted in a statistically significant difference in the electrode target and entry point errors. The Leksell frame seemed to be more stable than the Mayfield clamp in subjective experience during clinical use. In addition, we noticed that the target errors of some electrodes were smaller than the entry errors, which may be due to a rotation offset along the center of the brain caused by the registration.

\section{Limitations}

Limitations still exist in the present study. The sample that we evaluated was from a single center, which may have resulted in selection bias. Moreover, as SEEG electrodes were implanted based on a frameless stereotactic robotassisted system, the reference error, including the lateral, longitudinal and rotary deviation, could not be obtained for analysis. These deviations were finally shown as the entrance error, angular deviation and radial error. However, due to recent studies, the reference error is becoming very low. Additionally, we did not compare the accuracy between different stereotactic systems. A further study to analyze SEEG accuracy based on different stereotactic systems may be performed if necessary.

\section{Conclusions}

According to the present study, multilobe-spanning SEEG electrode implantation is a safe procedure with acceptable accuracy. The entrance error, depth error, and angular deviation are the primary influencing factors of the electrode target error, and the choice of head holder, the use of a predrill procedure and the angular deviation are the primary influencing factors of the electrode entrance error. The use of a predrill procedure could significantly reduce the electrode angular deviation. Further study should focus on modification of these influencing factors to enhance the accuracy of SEEG electrode implantation.

\section{Acknowledgments}

Funding: This work was supported by the National Natural Science Foundation of China (Grant Numbers 81801288 and 81871009). 


\section{Footnote}

Reporting Checklist: The authors have completed the STROBE reporting checklist. Available at http://dx.doi. org/10.21037/apm-20-2123

Data Sharing Statement: Available at http://dx.doi. org/10.21037/apm-20-2123

Conflicts of Interest: All authors have completed the ICMJE uniform disclosure form (available at http://dx.doi. org/10.21037/apm-20-2123). The authors have no conflicts of interest to declare.

Ethical Statement: The authors are accountable for all aspects of the work in ensuring that questions related to the accuracy or integrity of any part of the work are appropriately investigated and resolved. The study was conducted in accordance with the Declaration of Helsinki (as revised in 2013). The study was approved by the ethics committee at Xuanwu Hospital (No. LYS [2019] 097) and informed consent was taken from all the patients.

Open Access Statement: This is an Open Access article distributed in accordance with the Creative Commons Attribution-NonCommercial-NoDerivs 4.0 International License (CC BY-NC-ND 4.0), which permits the noncommercial replication and distribution of the article with the strict proviso that no changes or edits are made and the original work is properly cited (including links to both the formal publication through the relevant DOI and the license). See: https://creativecommons.org/licenses/by-nc-nd/4.0/.

\section{References}

1. Minotti L, Montavont A, Scholly J, et al. Indications and limits of stereoelectroencephalography (SEEG). Neurophysiol Clin 2018;48:15-24.

2. snard J, Taussig D, Bartolomei F, et al. French guidelines on stereoelectroencephalography (SEEG). Neurophysiol Clin 2018;48:5-13.

3. David O, Blauwblomme T, Job AS, et al. Imaging the seizure onset zone with stereo-electroencephalography. Brain 2011;134:2898-911.

4. Bottan JS, Rubino PA, Lau JC, et al. Robot-Assisted Insular Depth Electrode Implantation Through Oblique Trajectories: 3-Dimensional Anatomical Nuances, Technique, Accuracy, and Safety. Oper Neurosurg (Hagerstown) 2020;18:278-83.
5. Fan X, Shan Y, Lu C, et al. Optimized SEEG-guided radiofrequency thermocoagulation for mesial temporal lobe epilepsy with hippocampal sclerosis. Seizure 2019;71:304-11.

6. Wei PH, An Y, Fan XT, et al. StereoelectroencephalographyGuided Radiofrequency Thermocoagulation for Hypothalamic Hamartomas: Preliminary Evidence. World Neurosurg 2018;114:e1073-8.

7. Hou Z, Chen X, Shi XJ, et al. Comparison of Neuronavigation and Frame-Based Stereotactic Systems in Implanting Epileptic Depth Electrodes. Turk Neurosurg 2016;26:574-81.

8. Cardinale F, Cossu M, Castana L, et al. Stereoelectroencephalography: surgical methodology, safety, and stereotactic application accuracy in 500 procedures. Neurosurgery 2013;72:353-66; discussion 366.

9. Iordanou JC, Camara D, Ghatan S, et al. Approach Angle Affects Accuracy in Robotic Stereoelectroencephalography Lead Placement. World Neurosurg 2019;128:e322-8.

10. Mullin JP, Shriver M, Alomar S, et al. Is SEEG safe? A systematic review and meta-analysis of stereoelectroencephalography-related complications. Epilepsia 2016;57:386-401.

11. Girgis F, Ovruchesky E, Kennedy J, et al. Superior accuracy and precision of SEEG electrode insertion with frame-based vs. frameless stereotaxy methods. Acta Neurochir (Wien) 2020;162:2527-32.

12. González-Martínez J, Bulacio J, Thompson S, et al. Technique, Results, and Complications Related to RobotAssisted Stereoelectroencephalography. Neurosurgery 2016;78:169-80.

13. Brandmeir NJ, Savaliya S, Rohatgi $P$, et al. The comparative accuracy of the ROSA stereotactic robot across a wide range of clinical applications and registration techniques. J Robot Surg 2018;12:157-63.

14. Lefranc M, Capel C, Pruvot AS, et al. The impact of the reference imaging modality, registration method and intraoperative flat-panel computed tomography on the accuracy of the ROSA $\AA$ stereotactic robot. Stereotact Funct Neurosurg 2014;92:242-50.

Cite this article as: Lu C, Chen S, An Y, Fei M, Wang Y, Wei P, Fan X, Shan Y, Zhao G. How can the accuracy of SEEG be increased? - an analysis of the accuracy of multilobe-spanning SEEG electrodes based on a frameless stereotactic robotassisted system. Ann Palliat Med 2021;10(4):3699-3705. doi 10.21037/apm-20-2123 